\title{
Altitude effects of localized source currents on magnetotelluric responses
}

Shinya Sato ${ }^{*}$ (10

\begin{abstract}
The effects of localized source currents on Earth's magnetotelluric (MT) responses have been reported in the literature in terms of the changes in period and subsurface structure. The focus in this study is on the bias within the MT responses arising from variations in the vertical and horizontal distances of the source current. The MT responses at the periods of field aligned resonance (20 and $200 \mathrm{~s}$ ) were calculated at various distances from the source current. A slight change in source distance causes a shift in the MT responses. The shift of apparent resistivity at 20 and $200 \mathrm{~s}$ exceeds $10 \%$ and $50 \%$ of the original values, respectively. Such features are found especially over the altitude range of the ionospheric E layer (i.e., 100-150 km), where MT source currents often flow. The vertical distance of the source field varies because the distribution of conductivity with altitude in the ionosphere and the region controlling the ionospheric electrical process change temporally. Thus, in assessing the temporal changes in MT responses, we should treat them carefully by checking the ionospheric environment.
\end{abstract}

Keywords: Source bias, Magnetotelluric responses, Localized source currents

\section{Introduction}

In magnetotelluric (MT) surveys, the primary source magnetic fields are assumed horizontally uniform. The effects of localized source currents on the MT responses have been discussed in the literature (Madden and Nelson 1964; Schmucker 1970; Hermance and Peltier 1970; Häkkinen et al. 1989; Pirjola 1992; Viljanen 2012), where impedances over long periods and at sites above structures of high resistivity are biased because of the collapse of the plane-wave assumption. For example, Pirjola (1992) calculated the downward/upward bias of the apparent resistivities in the range of $1-100,000 \mathrm{~s}$ due to the electrojet with field-aligned currents under the condition that the divergence of the total current density vanishes. The study reported that the apparent resistivity of $100 \Omega \mathrm{m}$ and at periods larger than $60 \mathrm{~s}$ was clearly affected. Although past studies focused on perioddependent bias, the bias stemming from the variation

*Correspondence: sato.shinya.27s@st.kyoto-u.ac.jp Graduate School of Engineering, Kyoto University, C1-2 Kyoto

Daigaku-katsura, Nishikyo-ku, Kyoto 615-8540, Japan in distances, especially altitudes, of the localized source currents was not discussed in detail.

Temporal/seasonal changes (Brändlein et al. 2012; Romano et al. 2014; Vargas and Ritter 2016) and bias (Murphy and Egbert 2018) in the MT responses/vertical geomagnetic transfer-functions due to the source field have been recently reported. For example, Romano et al. (2014) reported that, for time periods $20-100 \mathrm{~s}$, the apparent resistivities have a negative correlation with geomagnetic activity. In particular, during time-lapse MT soundings, the source bias on the MT responses should be evaluated because we possibly misinterpret the temporal shifts in impedances of the source field as reflecting changes in the subsurface resistivity.

In this study, the electromagnetic fields and MT responses were calculated by varying the vertical and horizontal distances of the source current. The study revealed (i) the numerical examples of the bias in the MT responses because of the variation in vertical and horizontal distances of the source field, (ii) implications from these examples, (iii) the mathematical underpinning
Springer Open

(c) The Author(s) 2020. This article is licensed under a Creative Commons Attribution 4.0 International License, which permits use, sharing, adaptation, distribution and reproduction in any medium or format, as long as you give appropriate credit to the original author(s) and the source, provide a link to the Creative Commons licence, and indicate if changes were made. The images or other third party material in this article are included in the article's Creative Commons licence, unless indicated otherwise in a credit line to the material. If material. is not included in the article's Creative Commons licence and your intended use is not permitted by statutory regulation or exceeds the permitted use, you will need to obtain permission directly from the copyright holder. To view a copy of this licence, visit http://creativeco mmons.org/licenses/by/4.0/. 
of this bias, and (iv) the mathematical conditions for upholding the plane-wave assumption.

\section{Electromagnetic fields above Earth's surface}

We chose a Cartesian coordinate system, where the $x, y$, and $z$ axes are northward, westward, and downward positive, respectively, with $z=0$ at Earth's surface. The coordinate system, the line source current to be defined later, and an observation site are depicted in Fig. 1. Ignoring the displacement current and using the SI system, Maxwell's equations in the frequency domain are

$$
\begin{aligned}
& \nabla \times \mathbf{E}=-i \omega \mathbf{B}, \\
& \nabla \times \mathbf{B}=\mu_{0}(\sigma \mathbf{E}+\mathbf{J}), \\
& \nabla \cdot \mathbf{B}=0,
\end{aligned}
$$

where $\mathbf{E}, \mathbf{B}$, and $\mathbf{J}$ are the electric field, magnetic induction, and source current, respectively; $\sigma, \mu_{0}$, and $\omega$ are the electrical conductivity, the magnetic permeability of free space, and the angular frequency, respectively. Introducing the vector potential $\mathbf{A}$ and the scalar potential $\Pi$, the electromagnetic fields are

$$
\begin{aligned}
& \mathbf{B}=\nabla \times \mathbf{A}, \\
& \mathbf{E}=-i \omega(\mathbf{A}+\nabla \Pi) .
\end{aligned}
$$

We apply a gauge transformation such that $\mathbf{A}$ and $\Pi$ satisfy

$$
i \omega \sigma \mu_{0} \Pi=-\nabla \cdot \mathbf{A}
$$

and the equation for the vector potential can be represented as

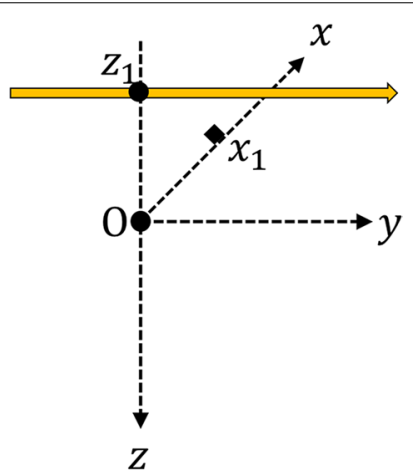

Fig. 1 Coordinate system used in this study. The symbol of "O" and diamond symbols mark the origin and an observation site, respectively; $z_{1}$ and $x_{1}$ are the altitude of the line source current and the horizontal distance of an observed site, respectively

$$
-\Delta \mathbf{A}+i \omega \sigma \mu_{0} \mathbf{A}=\mu_{0} \mathbf{J}
$$

Because $\nabla \cdot \mathbf{E}=0$, the equation for the scalar potential,

$$
-\Delta \Pi+i \omega \sigma \mu_{0} \Pi=0,
$$

holds. Considering the electromagnetic fields above Earth's surface (i.e., $z \leq 0$ ), $\sigma$ may be taken as $\sigma_{0}$ denoting the electrical conductivity of free space.

As in the analysis in Hermance and Peltier (1970), we consider a wire at an altitude $z_{1}<0$ carrying an electric current $I$ (Fig. 1); the current density is

$$
\mathbf{J}=\left(\begin{array}{c}
J_{x} \\
J_{y} \\
J_{z}
\end{array}\right)=I \delta\left(z-z_{1}\right) \delta(x)\left(\begin{array}{l}
0 \\
1 \\
0
\end{array}\right) .
$$

We focus on only $A_{y}$, the $y$ component of $\mathbf{A}$, and $\Pi$. For this study, the application of the Fourier transform (FT) to the horizontal components transforms $x$ and $y$ into the wavenumber domain yielding

$$
\begin{aligned}
& \tilde{F}(\eta, \zeta)=\int_{-\infty}^{\infty} \int_{-\infty}^{\infty} F(x, y) e^{i(\eta x+\zeta y)} d x d y \\
& F(x, y)=\frac{1}{4 \pi^{2}} \int_{-\infty}^{\infty} \int_{-\infty}^{\infty} \tilde{F}(\eta, \zeta) e^{-i(\eta x+\zeta y)} d \eta d \zeta
\end{aligned}
$$

Equation 11 enables us to transform Eq. 7 into

$$
\frac{\partial}{\partial z^{2}} \tilde{A}_{y}-\beta_{0}^{2} \tilde{A}_{y}=-\mu_{0} \tilde{J}_{y}(z \leq 0),
$$

where $\tilde{A}_{y}$ and $\tilde{J}_{y}$ are $A_{y}$ and $J_{y}$ in the wavenumber domain, respectively, and $\beta_{0}=\sqrt{\left(\eta^{2}+\zeta^{2}\right)+i \omega \mu_{0} \sigma_{0}}$. Equation 12 is the Helmholtz equation and for which its Green's function satisfies

$$
\frac{\partial}{\partial z^{2}} G\left(z, z^{\prime}\right)-\beta_{0}^{2} G\left(z, z^{\prime}\right)=\delta\left(z-z^{\prime}\right) .
$$

As shown in Arfken et al. (2012), the solution of $G\left(z, z^{\prime}\right)$ is

$$
G\left(z, z^{\prime}\right)=-\frac{\left(e^{-\beta_{0}\left|z-z^{\prime}\right|}+\Lambda e^{-\beta_{0}\left|z+z^{\prime}\right|}\right)}{2 \beta_{0}},
$$

where $\Lambda$ is a constant required to uphold the boundary condition at $z=0$. Consider a structure beneath Earth's surface $(z>0)$ having a half-space of conductivity $\sigma_{1}$. The continuity of the electromagnetic fields parallel to the boundary yields

$$
\Lambda(\eta, \zeta)=\frac{\beta_{0}-\beta_{1}}{\beta_{0}+\beta_{1}}
$$


where $\beta_{1}=\sqrt{\left(\eta^{2}+\zeta^{2}\right)+i \omega \mu_{0} \sigma_{1}}$. Applying the FT to the horizontal components, Eq. 10, $\tilde{J}_{y}$ in Eq. 12 becomes

$$
\tilde{J}_{y}=2 \pi I \delta\left(z-z_{1}\right) \delta(\zeta) .
$$

Using Green's function, the solutions for $\tilde{A}_{y}$ are

$$
\tilde{A}_{y}=\pi \mu_{0} I \frac{\left(e^{-\beta_{0}\left|z-z_{1}\right|}+\Lambda e^{-\beta_{0}\left|z+z_{1}\right|}\right) \delta(\zeta)}{\beta_{0}} .
$$

Similarly, applying the inverse FT to the horizontal components, Eq. 11, and considering $\sigma_{0}=0, A_{y}$ is written as

$$
A_{y}=\frac{\mu_{0} I}{4 \pi} \int_{-\infty}^{\infty} \frac{\left(e^{-|\eta|\left(z-z_{1}\right)}+\Lambda(\eta, 0) e^{|\eta|\left(z+z_{1}\right)}\right)}{|\eta|} e^{-i \eta x} d \eta(z \leq 0) .
$$

The vector potential A has only a $y$-component and $A_{y}$ is independent of $y$. As a result, the divergence of $\mathbf{A}$ is equal to zero, and the scalar potential is ignored because both sides of Eq. 6 vanish. From Eqs. 4 and 5, the magnetic induction $B_{x}$ and the electric field $E_{y}$ of a site $\left(x=x_{1}\right)$ at Earth's surface $(z=0)$ are written as

$$
\begin{aligned}
& B_{x}=\frac{\mu_{0} I}{2 \pi} \int_{-\infty}^{\infty} \frac{\sqrt{\eta^{2}+i \omega \mu_{0} \sigma_{1}}}{|\eta|+\sqrt{\eta^{2}+i \omega \mu_{0} \sigma_{1}}} e^{|\eta| z_{1}} e^{-i \eta x_{1}} d \eta, \\
& E_{y}=-i \omega \frac{\mu_{0} I}{2 \pi} \int_{-\infty}^{\infty} \frac{1}{|\eta|+\sqrt{\eta^{2}+i \omega \mu_{0} \sigma_{1}}} e^{|\eta| z_{1}} e^{-i \eta x_{1}} d \eta .
\end{aligned}
$$

Taking their ratio $E_{y} / B_{x}$ gives the impedance $Z_{y x}$

$$
Z_{y x}=\frac{-i \omega \int_{-\infty}^{\infty} \frac{1}{|\eta|+\sqrt{\eta^{2}+i \omega \mu_{0} \sigma_{1}}} e^{|\eta| z_{1}} e^{-i \eta x_{1}} d \eta}{\int_{-\infty}^{\infty} \frac{\sqrt{\eta^{2}+i \omega \mu_{0} \sigma_{1}}}{|\eta|+\sqrt{\eta^{2}+i \omega \mu_{0} \sigma_{1}}} e^{|\eta| z_{1}} e^{-i \eta x_{1}} d \eta} .
$$

For this study, the apparent resistivity $\rho_{y x}$ is given by

$$
\rho_{y x}=\frac{\mu_{0}}{2 \pi f}\left|Z_{y x}\right|^{2},
$$

where $Z_{y x}$ is defined in Eq. 21. Note that hereafter, for simplicity, $x_{1}$ is defined as the horizontal distance to the source current; the distance unit used is " $\mathrm{km}$ " instead of " $\mathrm{m}$ " when stating horizontal/vertical distances $\left(x_{1}, z_{1}\right)$ although all the above values are calculated using the SI system of units.

\section{MT responses biased by line source current}

Here, the subsurface resistivity and the time period are set to $1000 \Omega \mathrm{m}$ (i.e., $\sigma_{1}=10^{-3} \mathrm{~S} / \mathrm{m}$ ) and $20 \mathrm{~s}$, respectively. The subsurface resistivity has the same value as that used for the crust in Hermance and Peltier (1970).

By changing the altitude of the source current $z_{1}$ from -100 to $-1000 \mathrm{~km}$ in increments of $5 \mathrm{~km}$ and the horizontal distances $x_{1}=1,5,10,50,100,500,1000$, and $5000 \mathrm{~km}$, the variation in the field components $B_{x}$ (Eq. 19) and $E_{y}$ (Eq. 20) as well as the impedance $Z_{y x}$ (Eq. 21) were determined. The integrals in Eqs. 19 and 20 were calculated using the discrete approximation; the convergence of each was verified. Note that the electric current $I$ in Eq. 9 is 1000 A although the value has no influence on $Z_{y x}$. The calculated values of the apparent resistivity (Eq. 22) and phase are shown in Fig. 2. In addition, the values of the apparent
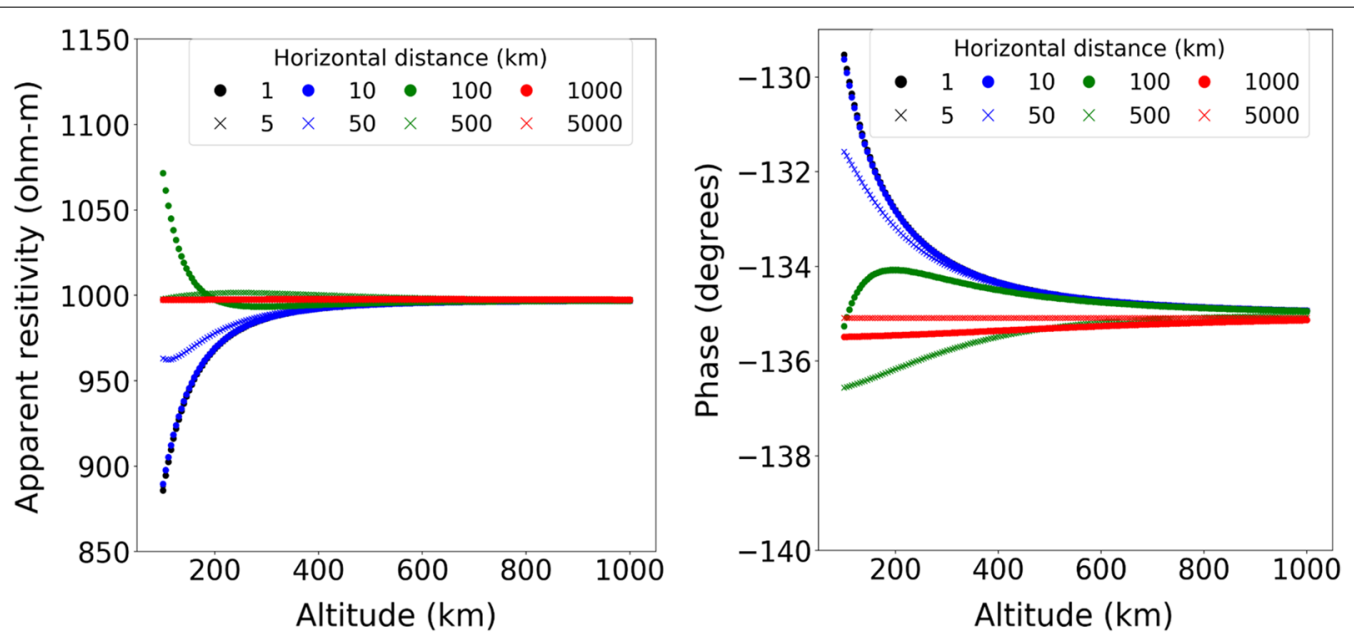

Fig. 2 MT responses at a period of $20 \mathrm{~s}$ : (left) relationship between the apparent resistivity and the altitude of the source current. (right) Relationship between the phase and the altitude of source current. The black/blue/green/red circles denote the responses derived from the horizontal distances of 1/10/100/1000 km, respectively. The black/blue/green/red crosses denote the responses derived from the horizontal distances of 5/50/500/5000 km, respectively 
resistivity and phase are derived by varying $x_{1}$ from 0 to $3000 \mathrm{~km}$ in increments of $10 \mathrm{~km}$ and $z_{1}$ set to $z_{1}=-100,-150,-200,-500$, and $-1000 \mathrm{~km}$ (Fig. 3). Based on these results (Figs. 2 and 3), the MT responses are shifted largely depending on the vertical and horizontal distances from the source current, if the altitude is within the range $100-150 \mathrm{~km}$ where the $\mathrm{E}$ region (Viljanen 2012; Sheng et al. 2014) with an important current system for MT exists. However, if $x_{1}$ and $\left|z_{1}\right|$ are larger, such bias becomes smaller.

The MT responses at a time period of $200 \mathrm{~s}$ under the same conditions as the above were plotted (Figs. 4 and 5) and as expected, are biased by the source current more than those of Figs. 2 and 3.

Results from lower resistivity values $(100 / 500 \Omega \mathrm{m})$ are presented in Additional file 1: Figs. S1-S8. These responses are less biased than the above cases (Figs. 2, 3, $4,5)$ as reported in the literature. If the conductivity and period are set to $0.01 \mathrm{~S} / \mathrm{m}$ and $20 \mathrm{~s}$, respectively, the bias within the MT responses approximately vanishes (Additional file 1: Figs. S1 and S2). However, the other cases (Additional file 1: Figs. S3-S8) are biased, especially if the altitude of the source current is within 100-150 km.

The line source current defined by Eq. 9 is possibly unrealistic, and maybe a sheet current is more suitable
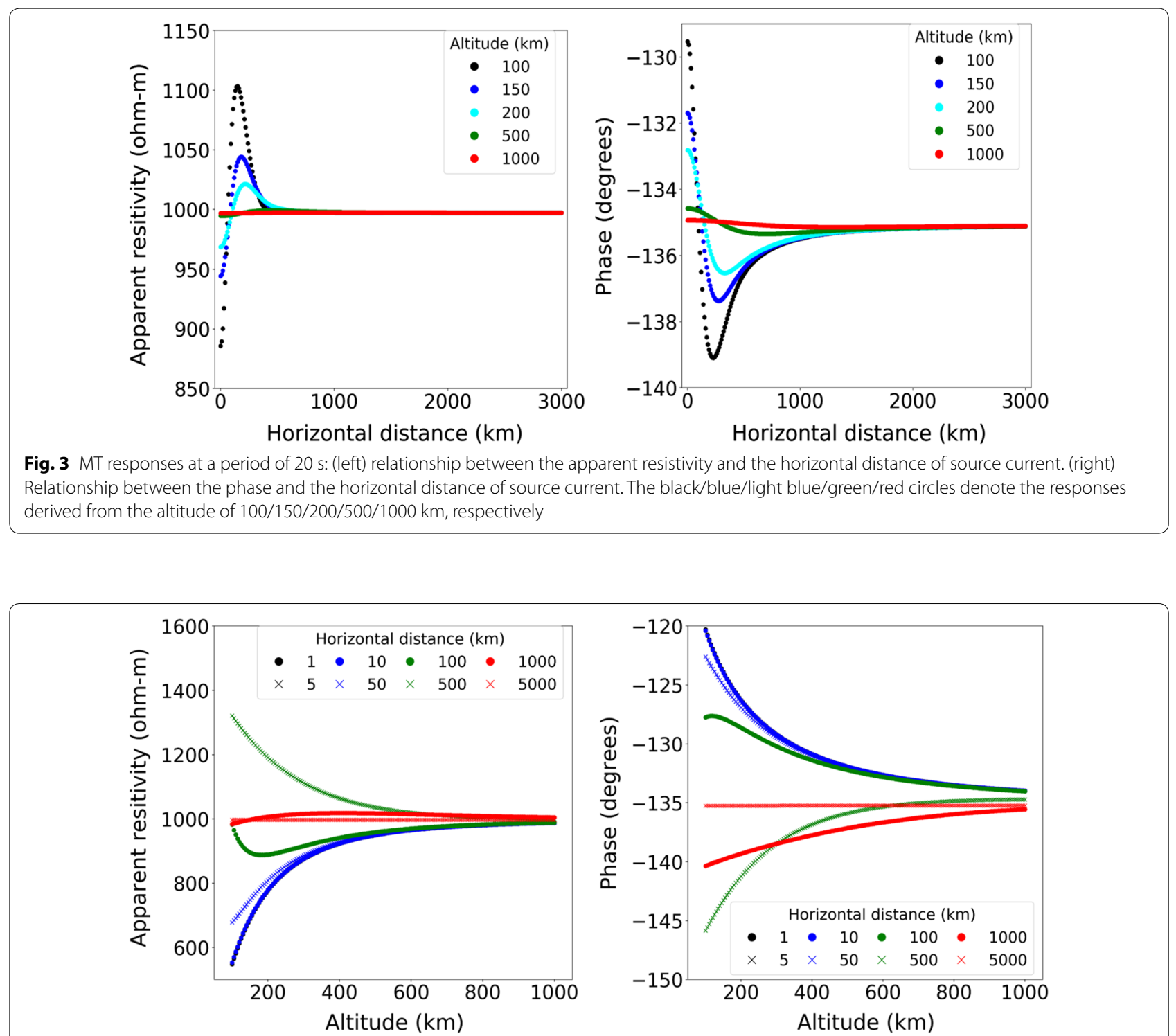

Fig. 4 As for Fig. 2 except the period is set to $200 \mathrm{~s}$ 

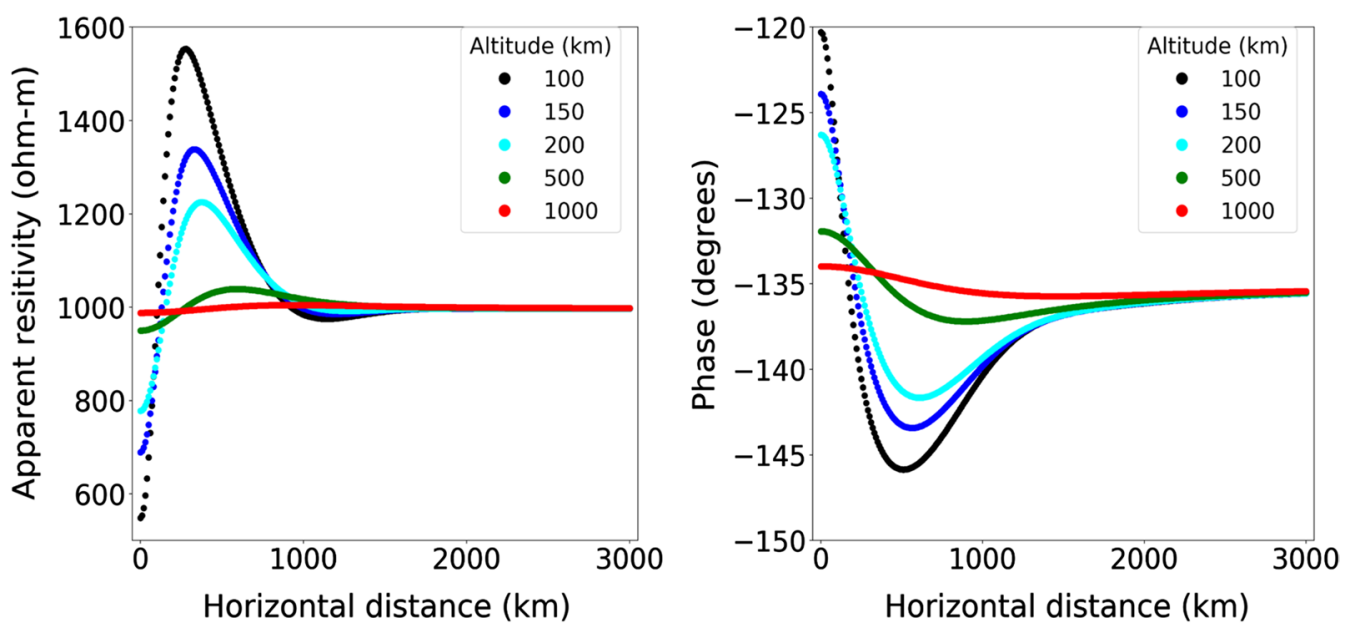

Fig. 5 As for Fig. 3 except the period is set to $200 \mathrm{~s}$

for actual current systems. The ionospheric current at mid-latitudes seems uniform over a wide area (Yamazaki and Maute 2017). However, the current system for example at low latitudes is controlled by an equatorial electrojet (Yamazaki and Maute 2017), which appears as a broad sheet current. As reported by McNish (1938), such current at altitudes higher than $100 \mathrm{~km}$ can be approximated by a line source current. Therefore, the entire analysis above, which has been derived assuming a line source current, is applicable to phenomena at low latitudes because in this study the altitude of the line current is at or above $100 \mathrm{~km}$.

\section{Discussion}

A discussion is presented next of (i) the mathematical basis of the bias on the MT responses due to the source field, (ii) the mathematical condition upholding the plane-wave assumption, and (iii) the implication arising from the numerical examples performed in this study.

The electromagnetic fields (Eqs. 19 and 20) generated by the line source current have an attenuation term

$$
\alpha(\eta)=e^{|\eta| z_{1}},
$$

and a term conveying information regarding the subsurface structure

$$
\beta(\eta)=\sqrt{\eta^{2}+i \omega \mu_{0} \sigma_{1}} .
$$

Substituting $\frac{2 \pi}{20} 1 / \mathrm{s}, 1.26 \cdot 10^{-6} \mathrm{H} / \mathrm{m}$, and $0.001 \mathrm{~S} / \mathrm{m}$ for $\omega, \mu_{0}$, and $\sigma_{1}$, respectively, the apparent resistivity and phase were plotted (Figs. 2 and 3). When the wavenumber $|\eta|$ is greater than $2.0 \cdot 10^{-5}$, the influence of $\eta$ is greater than $\omega \mu_{0} \sigma_{1}$. If $|\eta|$ vanishes, $\alpha(\eta)=1$ and the wavenumber effect on $\beta(\eta)$ vanishes. Hence, we set $\alpha(\eta)=1$ for the standard. When $\alpha(\eta)$ in Eq. 23 is smaller than 0.01, the effect of $|\eta|$ is assumed negligible because the attenuation term $\alpha(\eta)$ is two order of magnitude smaller than the standard (i.e., $\alpha(\eta)=1$ ). To uphold this assumption, $|\eta| z_{1}$ should be smaller than -4.6 , and when $z_{1}=-100 \mathrm{~km}$, $|\eta|$ should be greater than $4.6 \cdot 10^{-5}$. Therefore, the integrands in Eqs. 19 and 20 are biased by wavenumber $|\eta|$ at least within the interval $2.0 \cdot 10^{-5}<|\eta|<4.6 \cdot 10^{-5}$. However, given that $z_{1}=-1000 \mathrm{~km}, \alpha(\eta) \leq 2.1 \cdot 10^{-9}$ when substituting $|\eta| \geq 2.0 \cdot 10^{-5}$, which yields a greater effect than $\omega \mu_{0} \sigma_{1}$. The wavenumber effect is small enough to be negligible, and as a result, the apparent resistivity approaches a constant value of $1000 \Omega \mathrm{m}$, i.e., the subsurface resistivity. As expected from the above discussion, the MT responses at $200 \mathrm{~s}$ are biased more than those at $20 \mathrm{~s}$ because the influence of $\eta$ is greater than $\omega \mu_{0} \sigma_{1}$ when $|\eta|$ is greater than $6.3 \cdot 10^{-6}$. Moreover, on the basis of the above discussion, the MT responses at a site above a less-resistive zone can be expected to be less biased (see Additional file 1: Figs S1-S8). For example, suppose the conductivity and period are set to 0.01 S/m (i.e., $100 \Omega \mathrm{m}$ ) and $200 \mathrm{~s}$, respectively, as in Additional file 1: Figs. S5 and S6. Then, $\omega \mu_{0} \sigma_{1}$ in Eq. 24 calculated from these two variables has the same value as that obtained substituting $0.001 \mathrm{~S} / \mathrm{m}$ and $\frac{2 \pi}{20} 1 / \mathrm{s}$ (i.e., $20 \mathrm{~s}$ ) into $\sigma_{1}$ and $\omega$. As a result, both MT responses at $200 \mathrm{~s}$ (Additional file 1: Figs. S5 and S6) and at 20 s (Figs. 2 and 3) are shifted over a similar range (up to about $10 \%$ ).

Both responses at 20 and $200 \mathrm{~s}$ indicate the same patterns of bias; that is, they shift depending not only on the vertical $\left(z_{1}\right)$ but also on the horizontal distance $\left(x_{1}\right)$ between the site and the source current. The integrands in Eqs. 19 and 20 oscillate because of the factor $e^{-i \eta x_{1}}$, and substituting $5000 \mathrm{~km}$ into $x_{1}$, the apparent resistivity 
(Eq. 22) at $20 / 200 \mathrm{~s}$ has values of $1000 \Omega \mathrm{m}$ (see Figs. 2 and 4). The mathematics behind these calculated results resides with the re-expressions of Eqs. 19 and 20

$$
\begin{aligned}
& B_{x}=\frac{\mu_{0} I}{\pi} \int_{0}^{\infty} \frac{\sqrt{\eta^{2}+i a}}{\eta+\sqrt{\eta^{2}+i a}}\left(e^{\eta\left(z_{1}-i x_{1}\right)}+e^{\eta\left(z_{1}+i x_{1}\right)}\right) d \eta, \\
& E_{y}=-i \omega \frac{\mu_{0} I}{\pi} \int_{0}^{\infty} \frac{1}{\eta+\sqrt{\eta^{2}+i a}}\left(e^{\eta\left(z_{1}-i x_{1}\right)}+e^{\eta\left(z_{1}+i x_{1}\right)}\right) d \eta,
\end{aligned}
$$

where $a=\omega \mu_{0} \sigma_{1}$. Focusing on the term with $e^{\eta\left(z_{1}-i x_{1}\right)}$ and integrating by parts, we can obtain

$$
\begin{aligned}
& \int_{0}^{\infty} \frac{\sqrt{\eta^{2}+i a}}{\eta+\sqrt{\eta^{2}+i a}} e^{\eta\left(z_{1}-i x_{1}\right)} d \eta \\
&= \frac{-1}{\left(z_{1}-i x_{1}\right)}+\frac{-1}{\sqrt{i a}\left(z_{1}-i x_{1}\right)^{2}}+\frac{-2}{i a\left(z_{1}-i x_{1}\right)^{3}} \\
&-\int_{0}^{\infty} \frac{d^{3}}{d \eta^{3}}\left(\frac{\sqrt{\eta^{2}+i a}}{\eta+\sqrt{\eta^{2}+i a}}\right) \frac{e^{\eta\left(z_{1}-i x_{1}\right)}}{\left(z_{1}-i x_{1}\right)^{3}} d \eta \\
& \int_{0}^{\infty} \frac{e^{\eta\left(z_{1}-i x_{1}\right)}}{\eta+\sqrt{\eta^{2}+i a}} d \eta \\
&=\frac{-1}{\sqrt{i a}\left(z_{1}-i x_{1}\right)}+\frac{-1}{i a\left(z_{1}-i x_{1}\right)^{2}}+\frac{-1}{(i a)^{\frac{3}{2}}\left(z_{1}-i x_{1}\right)^{3}} . \\
&-\int_{0}^{\infty} \frac{d^{3}}{d \eta^{3}}\left(\frac{1}{\eta+\sqrt{\eta^{2}+i a}}\right) \frac{e^{\eta\left(z_{1}-i x_{1}\right)}}{\left(z_{1}-i x_{1}\right)^{3}} d \eta
\end{aligned}
$$

The triangle inequality and the inequality $\left|\frac{1}{\left(\eta^{2}+i a\right)}\right| \leq \frac{1}{a}(\eta \in \mathbb{R})$ enable the integrands of the last terms in Eqs. 27 and 28 to be examined

$$
\begin{aligned}
& \left|\int_{0}^{\infty} \frac{d^{3}}{d \eta^{3}}\left(\frac{\sqrt{\eta^{2}+i a}}{\eta+\sqrt{\eta^{2}+i a}}\right) e^{\eta\left(z_{1}-i x_{1}\right)} d \eta\right| \\
& \leq \int_{0}^{\infty} \frac{6 \eta+3 a}{a^{2}}\left(1+\frac{3 \eta^{2}+2 \sqrt{a} \eta}{a}\right) e^{\eta z_{1}} d \eta, \\
& \left|\int_{0}^{\infty} \frac{d^{3}}{d \eta^{3}}\left(\frac{1}{\eta+\sqrt{\eta^{2}+i a}}\right) e^{\eta\left(z_{1}-i x_{1}\right)} d \eta\right| \leq \int_{0}^{\infty} \frac{3 \eta}{a^{\frac{5}{2}}} e^{\eta z_{1}} d \eta .
\end{aligned}
$$

The right-hand sides of Eqs. 31 and 32 are constant and do not diverge. Replacing $\int_{0}^{\infty} \frac{d^{3}}{d \eta^{3}}\left(\frac{\sqrt{\eta^{2}+i a}}{\eta+\sqrt{\eta^{2}+i a}}\right) e^{\eta\left(z_{1}-i x_{1}\right)} d \eta$ and $\int_{0}^{\infty} \frac{d^{3}}{d \eta^{3}}\left(\frac{1}{\eta+\sqrt{\eta^{2}+i a}}\right) e^{\eta\left(z_{1}-i x_{1}\right)} d \eta$ with $C_{1}$ and $D_{1}$, respectively; the absolute values $\left|C_{1}\right|$ and $\left|D_{1}\right|$ are always less than a constant value. The same applies to $\int_{0}^{\infty} \frac{\sqrt{\eta^{2}+i a}}{\eta+\sqrt{\eta^{2}+i a}} e^{\eta\left(z_{1}+i x_{1}\right)} d \eta$ and $\int_{0}^{\infty} \frac{e^{\eta\left(z_{1}+i x_{1}\right)}}{\eta+\sqrt{\eta^{2}+i a}} d \eta$, with $\int_{0}^{\infty} \frac{d^{3}}{d \eta^{3}}\left(\frac{\sqrt{\eta^{2}+i a}}{\eta+\sqrt{\eta^{2}+i a}}\right) e^{\eta\left(z_{1}+i x_{1}\right)} d \eta$ and $\int_{0}^{\infty} \frac{d^{3}}{d \eta^{3}}\left(\frac{1}{\eta+\sqrt{\eta^{2}+i a}}\right) e^{\eta\left(z_{1}+i x_{1}\right)} d \eta$ being replaced by $C_{2}$ and $D_{2}$, respectively. As a result, the electromagnetic fields take the form

$$
\begin{aligned}
B_{x}= & -\frac{\mu_{0} I}{\pi}\left\{\frac{1}{\left(z_{1}-i x_{1}\right)}+\frac{1}{\sqrt{i a}\left(z_{1}-i x_{1}\right)^{2}}\right. \\
& +\frac{2+i a C_{1}}{i a\left(z_{1}-i x_{1}\right)^{3}}+\frac{1}{\left(z_{1}+i x_{1}\right)} \\
& \left.+\frac{1}{\sqrt{i a}\left(z_{1}+i x_{1}\right)^{2}}+\frac{2+i a C_{2}}{i a\left(z_{1}+i x_{1}\right)^{3}}\right\},
\end{aligned}
$$

$$
\begin{aligned}
\left|\frac{d^{3}}{d \eta^{3}}\left(\frac{\sqrt{\eta^{2}+i a}}{\eta+\sqrt{\eta^{2}+i a}}\right)\right|= & \mid \frac{3\left(\eta-\sqrt{\eta^{2}+i a}\right)}{\left(\eta+\sqrt{\eta^{2}+i a}\right)\left(\eta^{2}+i a\right)^{\frac{3}{2}}} \leq \frac{6 \eta+3 a}{a^{2}}\left(1+\frac{3 \eta^{2}+2 \sqrt{a} \eta}{a}\right), \\
& \left\{1+\frac{\eta\left(\eta+2 \sqrt{\eta^{2}+i a}\right)}{\left(\eta^{2}+i a\right)}\right\} \mid
\end{aligned}
$$

$$
\left|\frac{d^{3}}{d \eta^{3}}\left(\frac{1}{\eta+\sqrt{\eta^{2}+i a}}\right)\right|=\left|\frac{-3 \eta}{\left(\eta^{2}+a i\right)^{\frac{5}{2}}}\right| \leq \frac{3 \eta}{a^{\frac{5}{2}}} .
$$

Equations 29 and 30 and the inequality $\left|\int_{0}^{\infty} F(\eta) d \eta\right| \leq \int_{0}^{\infty}|F(\eta)| d \eta$, where $F$ is an arbitrary function, give

$$
\begin{aligned}
E_{y}= & i \omega \frac{\mu_{0} I}{\pi}\left\{\frac{1}{\sqrt{i a}\left(z_{1}-i x_{1}\right)}+\frac{1}{i a\left(z_{1}-i x_{1}\right)^{2}}\right. \\
& +\frac{1+(i a)^{\frac{3}{2}} D_{1}}{(i a)^{\frac{3}{2}}\left(z_{1}-i x_{1}\right)^{3}}+\frac{1}{\sqrt{i a}\left(z_{1}+i x_{1}\right)} \\
& \left.+\frac{1}{i a\left(z_{1}+i x_{1}\right)^{2}}+\frac{1+(i a)^{\frac{3}{2}} D_{2}}{(i a)^{\frac{3}{2}}\left(z_{1}+i x_{1}\right)^{3}}\right\} .
\end{aligned}
$$




$$
Z_{y x}=-i \omega \frac{\left\{\frac{1}{\sqrt{i a}\left(z_{1}-i x_{1}\right)}+\frac{1}{i a\left(z_{1}-i x_{1}\right)^{2}}+\frac{1+(i a)^{\frac{3}{2}} D_{1}}{(i a)^{\frac{3}{2}}\left(z_{1}-i x_{1}\right)^{3}}+\frac{1}{\sqrt{i a}\left(z_{1}+i x_{1}\right)}+\frac{1}{i a\left(z_{1}+i x_{1}\right)^{2}}+\frac{1+(i a)^{\frac{3}{2}} D_{2}}{(i a)^{\frac{3}{2}}\left(z_{1}+i x_{1}\right)^{3}}\right\}}{\left\{\frac{1}{\left(z_{1}-i x_{1}\right)}+\frac{1}{\sqrt{i a}\left(z_{1}-i x_{1}\right)^{2}}+\frac{2+i a C_{1}}{i a\left(z_{1}-i x_{1}\right)^{3}}+\frac{1}{\left(z_{1}+i x_{1}\right)}+\frac{1}{\sqrt{i a}\left(z_{1}+i x_{1}\right)^{2}}+\frac{2+i a C_{2}}{i a\left(z_{1}+i x_{1}\right)^{3}}\right\}} .
$$

In the limit $x_{1} \rightarrow \infty$, the plane-wave assumption is established

$$
Z_{y x}=-\frac{i \omega}{\sqrt{i a}}=\frac{-\sqrt{i \omega}}{\sqrt{\mu_{0} \sigma_{1}}}
$$

which is also upheld in the limit $z_{1} \rightarrow-\infty$. This means that if either the horizontal or vertical distance of the localized current is large enough, the plane-wave assumption remains valid. If this condition is not established, the MT responses would be biased by the source field (see Figs. 2, 3, 4, 5).

This study focused on the relationship between the MT responses and the vertical/horizontal distances of the source current. Given the numerical simulations (Figs. 2, 3, 4, 5 and Additional file 1: Figs. S1-S8) and the above discussions, the source bias diminishes if the altitude of the line source current rises, but becomes large especially when the altitude is within $100-150 \mathrm{~km}$. The altitude distribution of the conductivity in the ionosphere changes temporally/seasonally (Sheng et al. 2014). As shown in Maute and Richmond (2017), although the E layer controls the ionospheric electrical process during daytime, the $\mathrm{F}$ layer takes control during nighttime. Therefore, the altitude of the source current can be considered to vary temporally. Assuming the $\mathrm{E}$ and F layers are respectively at altitudes $100-150 \mathrm{~km}$ and 150 $600 \mathrm{~km}$ (Sheng et al. 2014), the MT data measured during nighttime possibly indicate a weakened source bias if we use only the line current in the ionosphere for a source. In addition, when deriving the MT responses using many spectra (i.e., long time-series data), this source bias may be neglected because the electromagnetic fields generated by the localized current are averaged. However, in the estimation of MT impedances from short-term data, the source effect must be considered because the electrical environment in the ionosphere mentioned above changes over a period of several hours to several months. Romano et al. (2014) observed source-dependent temporal changes in the MT responses even at mid-latitudes, where the current system is more likely to be uniform over a wide range than at low latitudes. Therefore, for time-lapse MT, especially at a site above the resistive structure (e.g., $1000 \Omega \mathrm{m}$ ) and at regions affected by a line source current (e.g., equatorial electrojet), the bias must be considered. Moreover, considering that the range in the biased apparent resistivities, having an initial value of $1000 \Omega \mathrm{m}$, is $550-1550 \Omega \mathrm{m}$ at $200 \mathrm{~s}$ (Fig. 5), the MT responses from short-term data must be used with care in the inversion procedure.

\section{Summary}

The focus in this study was on the bias in the MT responses arising from the variation in altitude and horizontal distances of a localized source current. The numerical examples show that slight changes in distance cause a shift in the MT response especially when the altitude is within $100-150 \mathrm{~km}$, where the E layer exists. These changes in MT responses are possibly seen in real MT data analysis because the vertical distance of the source current varies temporally/seasonally. Therefore, the bias in the MT responses for time-lapse sounding, especially at a region affected by a line source current (e.g., equatorial electrojet) and at a site above the resistive zone, should be evaluated to prevent such changes arising from the source field being regarded as resistivity changes in the subsurface. Moreover, considering that the range in shifts in the MT responses, especially at long periods, depends on source distance, we should treat these responses carefully, for example, by checking the ionospheric environment.

\section{Supplementary information}

Supplementary information accompanies this paper at https://doi. org/10.1186/s40623-020-01200-7.

Additional file 1. This additional file contains MT responses at periods of 20 s (Figs. S1-S4) and 200 s (Figs. S5-S8)obtained with resistivity values of 100 and $500 \Omega m$.

\section{Abbreviations}

MT: Magnetotelluric; FT: Fourier Transform.

\section{Acknowledgements}

The author thanks Dr. Tada-nori Goto, a professor at University of Hyogo, Dr. Katsuaki Koike, a professor at Kyoto University, and Mr. Fumihiko Onoue, a Ph.D. candidate at Scuola Normale Superiore di Pisa, for constructive comments. The author thanks two reviewers for a helpful review of the manuscript.

\section{Author's contributions}

The author read and approved the final manuscript.

Funding

This study is supported by MEXT (Ministry of Education, Culture, Sports, Science and Technology of Japan), through a Grant-in-Aid for Scientific Research (JSPS KAKENHI Grant Number JP18J20941). 
Availability of data and materials

Not applicable.

\section{Ethics approval and consent to participate}

Not applicable.

\section{Consent for publication}

Not applicable.

\section{Competing interests}

Not applicable.

Received: 4 March 2020 Accepted: 18 May 2020

Published online: 01 June 2020

\section{References}

Arfken GB, Weber HJ, Harris FE (2012) Mathematical methods for physicists: a comprehensive guide. Academic Press, Cambridge

Brändlein D, Lühr H, Ritter O (2012) Direct penetration of the interplanetary electric field to low geomagnetic latitudes and its effect on magnetotelluric sounding. J Geophys Res 117:A11. https://doi.org/10.1029/2012j a018008

Häkkinen L, Pirjola R, Sucksdorff C (1989) EISCAT magnetometer cross and theoretical studies connected with the electrojet current system. Geophysica 25(1):123-134

Hermance JF, Peltier WR (1970) Magnetotelluric fields of a line current. J Geophys Res 75(17):3351-3356

Madden T, Nelson P (1964) A defense of Cagniard's magnetotelluric method. ONR Rept. pp 371-401

Maute A, Richmond AD (2017) F-region dynamo simulations at low and midlatitude. Space Sci Rev 206:471-493
McNish AG (1938) Heights of electric currents near the Auroral Zone. Terr Magn Atmos Electr 43:67-75

Murphy BS, Egbert GD (2018) Source biases in midlatitude magnetotelluric transfer functions due to Pc3-4 geomagnetic pulsations. Earth Planets Space 70(1):12. https://doi.org/10.1186/s40623-018-0781-0

Pirjola R (1992) On magnetotelluric source effects caused by an auroral electrojet system. Radio Sci 27(04):463-468

Romano G, Balasco M, Lapenna V, Siniscalchi A, Telesca L, Tripaldi S (2014) On the sensitivity of long-term magnetotelluric monitoring in Southern Italy and source-dependent robust single station transfer function variability. Geophys J Int 197(3):1425-1441

Schmucker U (1970) Anomalies of geomagnetic variations in the southwestern United States. Bull Scripps Inst Oceanogr, Berkeley

Sheng C, Deng Y, Yue X, Huang Y (2014) Height-integrated Pedersen conductivity in both $E$ and $F$ regions from COSMIC observations. J Atmos Sol Terr Phys 115:79-86

Vargas JA, Ritter O (2016) Source effects in mid-latitude geomagnetic transfer functions. Geophys J Int 204(1):606-630

Viljanen A (2012) Description of the magnetospheric/ionospheric sources. In: Chave AD, Jones AG (eds) The magnetotelluric method. Cambridge University Press, Cambridge

Yamazaki Y, Maute A (2017) Sq and EEJ_-a review on the daily variation of the geomagnetic field caused by ionospheric dynamo currents. Space Sci Rev 206:299-405

\section{Publisher's Note}

Springer Nature remains neutral with regard to jurisdictional claims in published maps and institutional affiliations.

\section{Submit your manuscript to a SpringerOpen ${ }^{\circ}$ journal and benefit from:}

- Convenient online submission

- Rigorous peer review

- Open access: articles freely available online

- High visibility within the field

- Retaining the copyright to your article

Submit your next manuscript at $\boldsymbol{\nabla}$ springeropen.com 